\title{
DETERMINACIÓN DE LAS CARACTERÍSTICAS GEOMÉTRICAS DE LA CAÑA GUADUA ANGUSTIFOLIA DEL ECUADOR
}

\author{
DETERMINATION OF GEOMETRIC CHARACTERISTICS OF THE BAMBOO \\ GUADUA ANGUSTIFOLIA OF ECUADOR
}

\section{ANTONIO LA TEGOLA', LUIS OCTAVIO YÉPEZ ROCA², WALTER VICENTE MERA ORTIZ², PEDRO LUIS CÓRDOVA ALCÍVAR}

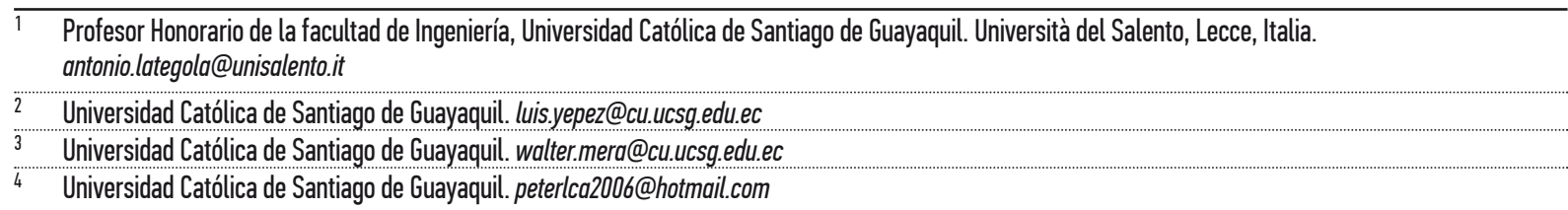

RESUMEN

El principal objetivo de este trabajo de investigación es la determinación de las características geométricas de la caña Guadua Angustifolia Kunth de Ecuador, y a través de la metodología de las mediciones determinar relaciones estadísticas entre sus características. Se tomaron medidas a tallos de caña guadua en diferentes depósitos y plantaciones de las provincias costeras del Ecuador. Estas se las efectuaron con calibrador vernier para tener precisión en la obtención de los valores de diámetro y espesores y se usó cinta métrica para obtener longitudes o distancia de separación de nudos (entrenudo). El conjunto de las variables será explicado a través del estudio de la información estadística. Los resultados permitieron determinar los valores característicos de las variables de estudio.

PALABRAS CLAVE: propiedades geométricas, caña guadua Angustifolia Kunth.
ABSTRACT

The main objective of this research is to determine the geometrical characteristics of the Bamboo Guadua Angustifolia Kunth of Ecuador, and through the methodology of measurements to determine statistical relationships between its characteristics. Culms were measured in different deposits and plantations of the coastal provinces of Ecuador. These were made with vernier caliper for precision in obtaining the values of diameter and thickness and tape measure to obtain lengths between nodes. The set of variables will be explained through the study of statistical information. The test results allowed to determine the characteristic values of the study variables.

KEYWORDS: geometric properties, bamboo guadua Angustifolia Kunth. 
INTRODUCCIÓN

Las variables a medir son las propiedades geométricas delbambú (caña guadua): diámetro exterior; diámetro interior; espesor de la pared de la caña; longitud de separación entre nudos; porcentaje de imperfección vertical de la caña guadua.

El objetivo del trabajo es deducir todos los parámetros a solo uno. Por lo tanto se hace referencia a la teoría probabilística para determinar los valores característicos.

De acuerdo a la metodología recomendada por La Tegola y Mera (2012), los valores característicos de la propiedad geométrica se calcularon como percentiles de propiedad menor que 5\%. Teniendo en cuenta el número de muestras, se hizo referencia a la teoría de pequeñas muestras, mediante la estimación de la media y de la varianza. Además se adoptó un intervalo de confianza igual al $75 \%$ con el fin de tener en cuenta la naturaleza del material.

\section{METODOLOGÍA}

Según Córdova (2014), las cañas de bambú de la variedad guadua constituyen una alternativa eficaz para la construcción de estructuras sismoresistentes y eco compatibles. Las cañas son utilizadas ya sea como elementos solicitados a compresión bajo la forma de columnas y de puntales y como elementos tipo vigas. Bajo el aspecto geométrico los parámetros necesarios para el cálculo son las dimensiones transversales, las longitudinales y las imperfecciones de las cañas.

Los parámetros transversales son el diámetro externo y el diámetro interno y por consiguiente el espesor de la sección anular hueca; los parámetros longitudinales son la distancia entre los nudos y la rectilinealidad por la presencia de curvatura.

Los resultados derivados de las investigaciones experimentales son analizados bajo el aspecto probabilístico determinándose todos los valores característicos con probabilidad al 5\%. Obviamente en la verificación de la seguridad deben ser introducidos los valores de cálculo de las resistencias y los valores de cálculo de las acciones. Toda la investigación experimental de tipo estadístico ha consistido en el considerar los varios parámetros como variables aleatorias asimilables a una distribución de tipo Gamma. La definición de los valores característicos es además tratada con la teoría de las muestras pequeñas, aplicando el Test de Student y del Chi-cuadrado en función de los intervalos de confianza adoptados.

Ya que las cañas, sea bajo el aspecto geométrico o mecánico, poseen índices de dispersión significativos, se considera que el intervalo de confianza sea igual al 75\% como aquel adoptado para otros materiales actualmente utilizados.

Por lo tanto, la definición de cualquier parámetro característico es validada según la relación: $A_{k}=A_{m} \pm(K)(s)$

Siendo $A_{k}=A_{m} \pm(K)(s)$ la media de la muestra

$$
\begin{gathered}
s=\sqrt{\frac{\Sigma(A(i)-A(m))^{2}}{N-1}} \text { la desviación estándar y } \\
K^{*}=K^{*}(\theta, p, N) \text { Función del intervalo de }
\end{gathered}
$$
confianza $\theta=0.75$ del fratil $\mathrm{p}=0.05 \mathrm{y}$ del número $\mathrm{N}$ de las muestras. Los valores de $\mathrm{K}^{*}$ son reportados en la Tabla 1.

TABLA 1. VALORES K DEL INTERVALO DE CONFIANZA EN FUNCIÓN DEL NÚMERO N DE LAS MUESTRAS

\begin{tabular}{rr}
$\mathbf{N}$ & $\mathbf{P}=\mathbf{0}, 75$ \\
& $\mathbf{p = 0 , 0 5}$ \\
6 & 2,68 \\
8 & 2,33 \\
10 & 2,19 \\
12 & 2,10 \\
14 & 2,05 \\
16 & 2,00 \\
18 & 1,97 \\
20 & 1,95 \\
25 & 1,93 \\
30 & 1,89 \\
40 & 1,86 \\
50 & 1,82 \\
60 & 1,79 \\
75 & 1,78 \\
100 & 1,76 \\
\hline 200 & 1,74 \\
500 & 1,71 \\
$\infty$ & 1,68 \\
\hline & 1,65 \\
\hline
\end{tabular}


PARÁMETROS GEOMÉTRICOS

Los parámetros geométricos transversales necesarios para el diseño son aquellos que permiten definir la sección, el momento de inercia, los módulos de resistencia a la flexión y a cortante, y el radio de giro.

Dichas cantidades dependen del diámetro externo y del diámetro interno y por consiguiente del espesor de la sección anular.

PARÁMETROS TRANSVERSALES

La primera investigación ha sido realizada con el fin de relacionar el espesor al diámetro externo determinando un parámetro característico. Con dicho fin se ha examinado 108 muestras, midiendo el diámetro externo $\mathrm{D}_{\mathrm{e}}$, el diámetro interno $\mathrm{D}_{\mathrm{i}}$ y por lo tanto el espesor:

$e_{s}=\frac{D_{e}-D_{i}}{2}$,y por consiguiente la relación $\partial_{s}=\frac{e_{s}}{D_{e}}$

Dichas 108 muestras han provisto:

$$
\partial_{s m}=\frac{\Sigma \partial_{s}(i)}{N}=0.138 \quad s=\sqrt{\frac{\Sigma\left(\partial_{s}(i)-\partial_{s m}\right)^{2}}{N-1}}=0.036
$$

Por lo tanto, teniendo en cuenta el número de muestras el valor característico de la relación $\partial_{s}=\frac{e_{s}}{D_{e}} \quad$ como fratil inferior al $5 \%$ está dado por:

$$
\partial_{s k}=\partial_{s m}-(k)(s)=0.138-(1.74)(0.036)=0.075
$$

Resulta por lo tanto $e_{s}=0.075 D_{e}$

Las cantidades geométricas que interesan son:

Sección:

$$
\begin{gathered}
A=\frac{\pi\left(D_{e}^{2}-D_{i}^{2}\right)}{4}=\frac{\pi\left(D_{e}-D_{i}\right)\left(D_{e}+D_{i}\right)}{4}=\pi e_{s} D_{m} \\
e_{s}=\delta_{s k} D_{e} \quad D_{m}=D_{e}-e_{s}
\end{gathered}
$$

Momento de Inercia:

$$
\begin{aligned}
& I=\frac{\pi\left(D_{e}^{4}-D_{i}^{4}\right)}{64}=\frac{\pi\left(D_{e}^{2}-D_{i}^{2}\right)\left(D_{e}^{2}+D_{i}^{2}\right)}{64}= \\
& =\frac{\pi\left(D_{e}-D_{i}\right)\left(D_{e}+D_{i}\right)\left(D_{e}^{2}+D_{i}^{2}\right)}{64}=\frac{\pi e_{s} D_{m}^{3}}{8}
\end{aligned}
$$

Momento de resistencia a flexión:

$$
W=\frac{\pi e_{s} D_{m}^{2}}{4}
$$

Módulo de resistencia al cortante:

$$
K=\frac{2 e_{S} I}{S}=\frac{2 W}{D_{m}}
$$

Radio de Inercia:

$$
r=\sqrt{\frac{I}{A}}=\frac{D_{m}}{2 \sqrt{2}}
$$

Todos los parámetros geométricos, para varios valores de $\mathrm{D}_{\mathrm{e}}$, son reportados en la Tabla 2, habiendo asumido: $e_{s}=0.075 D_{e}$

\section{TABLA 2. PARÁMETROS GEOMÉTRICOS DE CAÑAS GUADUA CON DIFERENTES VALORES DE DIÁMETRO EXTERNO

DE (CM) ES (CM) A (CM2) I (CM4) $\quad$ W (CM3) $\quad$ K (CM2) $\quad$ R (CM)

\begin{tabular}{lllllll}
\hline 8.00 & 0.60 & 13.95 & 95.48 & 25.81 & 6.97 & 2.62 \\
8.25 & 0.62 & 14.83 & 107.98 & 28.30 & 7.42 & 2.70 \\
8.50 & 0.64 & 15.75 & 121.58 & 30.95 & 7.87 & 2.78 \\
8.75 & 0.66 & 15.59 & 136.64 & 33.76 & 8.34 & 2.86 \\
\hline 9.00 & 0.68 & 17.65 & 152.94 & 36.74 & 8.83 & 2.94 \\
9.25 & 0.69 & 18.65 & 170.65 & 39.89 & 9.32 & 3.03
\end{tabular}

\begin{tabular}{rrrrrrr}
\hline 9.50 & 0.71 & 17.57 & 189.85 & 43.21 & 9.83 & 3.11 \\
\hline 9.75 & 0.73 & 20.72 & 210.55 & 46.71 & 10.36 & 3.19 \\
\hline 10.00 & 0.75 & 21.79 & 233.10 & 50.40 & 10.90 & 3.27 \\
\hline 10.25 & 0.77 & 22.90 & 257.30 & 54.28 & 11.45 & 3.35 \\
\hline 10.50 & 0.79 & 24.03 & 283.34 & 58.34 & 12.01 & 3.43 \\
\hline 10.75 & 0.81 & 25.19 & 311.30 & 62.61 & 12.59 & 3.52 \\
\hline 11.00 & 0.83 & 26.17 & 341.97 & 67.18 & 13.19 & 3.60 \\
\hline 11.25 & 0.84 & 27.58 & 373.39 & 71.76 & 13.79 & 3.68 \\
\hline 11.50 & 0.86 & 28.82 & 407.70 & 76.65 & 14.41 & 3.76 \\
\hline 11.75 & 0.88 & 30.09 & 444.32 & 81.76 & 15.05 & 3.84 \\
\hline 12.00 & 0.90 & 31.38 & 488.36 & 87.09 & 15.69 & 3.92 \\
\hline 12.25 & 0.92 & 32.71 & 524.92 & 92.65 & 16.35 & 4.01 \\
\hline 12.50 & 0.94 & 34.05 & 569.10 & 98.44 & 17.03 & 4.09 \\
\hline 12.75 & 0.96 & 35.43 & 616.01 & 104.46 & 17.72 & 4.17 \\
\hline 13.00 & 0.98 & 36.83 & 665.76 & 110.73 & 18.42 & 4.25 \\
\hline 13.25 & 0.99 & 38.26 & 718.17 & 117.24 & 19.13 & 4.33 \\
\hline 13.50 & 1.01 & 39.72 & 774.25 & 124.00 & 19.86 & 4.41 \\
\hline 13.75 & 1.03 & 41.21 & 833.22 & 131.02 & 20.60 & 4.50 \\
\hline 14.00 & 1.05 & 42.77 & 825.47 & 138.30 & 21.36 & 4.58 \\
\hline 14.25 & 1.07 & 44.26 & 961.18 & 145.84 & 22.13. & 4.66 \\
\hline 14.50 & 1.09 & 45.82 & 1030.43 & 153.65 & 22.91 & 4.74 \\
\hline 14.75 & 1.11 & 47.42 & 1103.36 & 161.74 & 23.71 & 4.82 \\
\hline $15 . .00$ & 1.13 & 49.04 & 1180.08 & 170.10 & 24.52 & 4.91 \\
\hline & & & & & & \\
\hline
\end{tabular}

PARÁMETROS LONGITUDINALES DISTANCIA INTERNODAL

La segunda investigación se ha desarrollado con el fin de relacionar la distancia entre los nudos (intermodal) al diámetro externo, determinándose también en este caso un parámetro característico. 
Se ha examinado una muestra de 189 cañas y se ha medido la distancia interior y se ha calculado la relación:

$$
\lambda_{\text {in }}=\frac{\text { Int }}{D_{2}}
$$

Se ha obtenido: $\quad \lambda_{\text {inm }}=\frac{\Sigma \lambda_{\text {in }}(i)}{N}=2.126$

$$
s=\sqrt{\frac{\sum\left(\lambda_{i n}(i)-\lambda_{i n m}\right)^{2}}{N-1}}=0.67
$$

Y haciendo referencia al fratil superior se obtiene:

$$
\lambda_{\text {ink }}=2.126+(1.71)(0.67)=3.272
$$

IMPERFECCIONES DE RECTILINEALIDAD

Una tercera investigación se ha realizado además para la determinación de la no perfecta linealidad de las cañas. Se han examinado 28 cañas de longitud y diámetros diferentes. Las imperfecciones en relación a la longitud de una caña simple han producido un valor en porcentaje.

Se ha obtenido un valor medio:

$$
i_{1 m m}=\frac{\Sigma i_{1 m}(i)}{N}=0.707 \%
$$

Una desviación estándar:

$$
s=\sqrt{\frac{\sum\left(i_{1 m}(i)-i_{1 m m}\right)^{2}}{N-1}}=0.316
$$

Y por tanto un valor característico:

$$
\begin{aligned}
& i_{1 \mathrm{mk}}=0.707+(1.87)(3.16)=1.298 \% \\
& \frac{s}{i_{1 \mathrm{~mm}}}=0.447
\end{aligned}
$$

Dichas imperfecciones son importantes ya que en las cañas solicitadas a compresión una excentricidad de primer orden puede generar excentricidades de segundo orden importantes que no pueden ser despreciados.

PESO PROPIO DEL BAMBÚ Y DE LAS CAÑAS

Se ha realizado una investigación análoga para determinar la densidad del material. Se han evaluado los siguientes valores:

$$
\begin{array}{lll}
\gamma_{m}=937 \frac{\mathrm{kg}}{\mathrm{m}^{3}} & \mathrm{~s}=215 & \gamma_{m}=1388 \frac{\mathrm{kg}}{\mathrm{m}^{3}} \\
\frac{\mathrm{s}}{\gamma_{m}}=0.229 & & \\
\frac{p_{m}}{D_{e}}=29 \frac{\mathrm{kg}}{\mathrm{m}} & \mathrm{s}=5 & \frac{p_{m k}}{D_{e}}=41 \frac{\mathrm{kg}}{\mathrm{m}} \\
\frac{\mathrm{s}}{\left(\frac{p_{m}}{D_{e}}\right)}=0.184 & \mathrm{~s}=2 & \gamma_{s k}=13 \frac{\mathrm{kg}}{\mathrm{m}^{2}}
\end{array}
$$

Densidad del material bambú $=1388 \frac{\mathrm{kg}}{\mathrm{m}^{3}}$

Peso de la caña bambú $=41 \times D_{e} \frac{k g}{m}\left(D_{e} e n m\right)$

Peso pared con caña abierta $=13 \frac{\mathrm{kg}}{\mathrm{m}^{2}}$

RESULTADOS

En las tablas 3 y 4 se muestran los resultados de mediciones hechas a 246 cañas de guadua de diversos orígenes de la costa ecuatoriana. Se midieron los diametros interno y externo, espesor de la pared de la caña y longitud de los entrenudos.

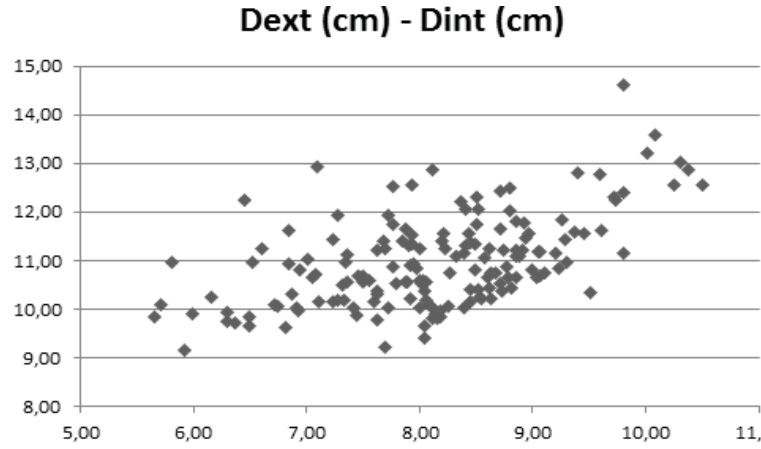

Figura 1. Variación del diámetro exterior de la caña guadua vs. el diámetro interior. A medida que aumenta el diámetro exterior aumenta el diámetro interior. Son directamente proporcionales.

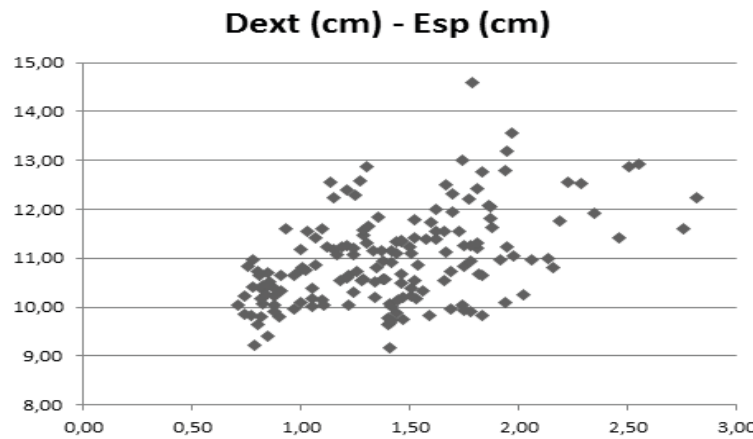

Figura 2. Variación del diámetro exterior de la caña guadua vs. el espesor de la pared de la caña. A medida que aumenta el diámetro exterior aumenta el espesor de la pared de la caña.

Dext (cm) - S. Int (cm)

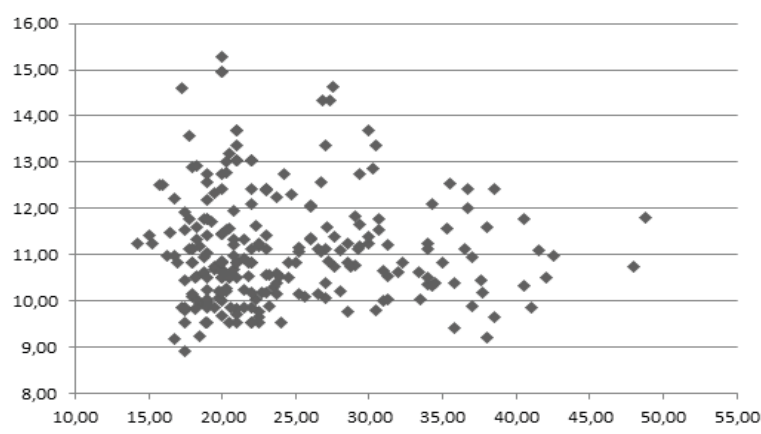

Figura 3. Variación del diámetro exterior de la caña guadua vs. la longitud de entrenudo. A medida que disminuye el diámetro exterior, aumenta la longitud del entrenudo. 
DETERMINACIÓN DE LAS CARACTERÍSTICAS GEOMÉTRICAS DE LA CAÑA GUADÚA ANGUSTIFOLIA DEL ECUADOR.

TABLA 3. PARÁMETROS GEOMÉtrICOS MEdIDOS A MUESTRAS dE CAÑA GUADUA ANGUSTIFOLIA KUNTH

\begin{tabular}{|c|c|c|c|c|c|c|c|c|c|c|c|c|c|c|}
\hline $\begin{array}{l}\text { MRO. DE } \\
\text { MUESTRA }\end{array}$ & $\begin{array}{r}\text { DIAMETRO } \\
\text { EXTERIOR } \\
\text { (CM) }\end{array}$ & $\begin{array}{r}\text { DIAMETRO } \\
\text { INTERIOR } \\
\text { (CM) }\end{array}$ & $\begin{array}{r}\text { ESPESOR } \\
\text { (CM) }\end{array}$ & $\begin{array}{r}\text { S. ITERNUDO } \\
\text { (CM) }\end{array}$ & $\begin{array}{l}\text { NRO. DE } \\
\text { MUESTRA }\end{array}$ & $\begin{array}{r}\text { DIAMETRO } \\
\text { EXTERIOR } \\
\text { (CM) }\end{array}$ & $\begin{array}{r}\text { DIAMETRO } \\
\text { INTERIOR } \\
\text { (CM) }\end{array}$ & $\begin{array}{r}\text { ESPESOR } \\
\text { (CM) }\end{array}$ & $\begin{array}{r}\text { S. ITERNUDO } \\
\text { (CM) }\end{array}$ & $\begin{array}{r}\text { MRO. DE } \\
\text { MUESTRA }\end{array}$ & $\begin{array}{r}\text { DIAMETRO } \\
\text { EXTERIOR } \\
\text { (CM) }\end{array}$ & $\begin{array}{r}\text { DIAMETRO } \\
\text { INTERIOR } \\
\text { (CM) }\end{array}$ & $\begin{array}{r}\text { ESPESOR } \\
\text { (CM) }\end{array}$ & $\begin{array}{r}\text { S. ITERNUDO } \\
\text { (CM) }\end{array}$ \\
\hline 1 & 10,18 & 8,06 & 1,05 & 37,75 & 56 & 12,88 & 8,12 & 2,51 & 18,00 & 111 & 9,78 & 7,63 & 1,40 & 22,50 \\
\hline 3 & 10,67 & 7,50 & 1,46 & 19,50 & 58 & 11,94 & 7,73 & 1,70 & 20,75 & 113 & 10,03 & 8,39 & 0,71 & 31,25 \\
\hline 4 & 10,26 & 6,16 & 2,02 & 20,25 & 59 & 11,64 & 7,88 & 1,88 & 22,25 & 114 & 10,97 & 6,53 & 2,06 & 16,75 \\
\hline 7 & 10,30 & 7,62 & 1,24 & 23,50 & 62 & 10,68 & 7,46 & 1,82 & 20,75 & 117 & 9,91 & 5,99 & 1,78 & 19,00 \\
\hline 8 & 10,15 & 7,60 & 1,10 & 25,25 & 63 & 12,40 & 9,80 & 1,21 & 23,00 & 118 & 10,82 & 8,49 & 1,35 & 25,00 \\
\hline 9 & 10,19 & 7,28 & 1,34 & 22,75 & 64 & 10,55 & 7,36 & 1,66 & 18,25 & 119 & 10,60 & 8,00 & 1,21 & 23,75 \\
\hline 10 & 9,00 & 6,93 & 1,69 & 18,50 & 65 & 11,54 & 7,93 & 1,73 & 17,50 & 120 & 10,57 & 7,50 & 1,39 & 20,25 \\
\hline 11 & 96,00 & 7,70 & 0,79 & 38,00 & 66 & 11,92 & 7,28 & 2,35 & 17,50 & 121 & 12,92 & 7,10 & 2,55 & 18,25 \\
\hline 15 & 12,76 & 9,80 & 1,79 & 17,25 & 70 & 11,10 & 7,36 & 1,67 & 17,75 & 125 & 12,30 & 9,72 & 1,25 & 24,75 \\
\hline 16 & 14,60 & 7,77 & 1,54 & 20,00 & 71 & 10,52 & 8,71 & 0,86 & 34,00 & 126 & 10,14 & 5,71 & 1,94 & 18,00 \\
\hline 17 & 10,86 & 9,10 & 0,80 & 48,00 & 72 & 11,55 & 8,74 & 1,12 & 20,75 & 127 & 11,37 & 8,45 & 1,46 & 26,08 \\
\hline 18 & 10,74 & 7,98 & 1,75 & 24,50 & 73 & 10,64 & 7,06 & 1,83 & 18,75 & 128 & 11,22 & 8,92 & 1,24 & 31,25 \\
\hline 19 & 10,84 & 8,52 & 0,78 & 34,50 & 74 & 12,25 & 9,74 & 1,15 & 23,67 & 129 & 11,84 & 9,26 & 1,36 & 29,00 \\
\hline 20 & 10,40 & 7,77 & 2,19 & 18,75 & 75 & 11,19 & 9,07 & 1,15 & 29,33 & 130 & 10,45 & 8,62 & 0,83 & 37,63 \\
\hline 21 & 11,76 & 7,08 & 1,69 & 19,50 & 76 & 11,78 & 8,93 & 1,52 & 17,75 & 131 & 11,60 & 9,37 & 1,10 & 27,13 \\
\hline 22 & 10,73 & 7,73 & 1,22 & 22,25 & 77 & 9,42 & 6,30 & 1,75 & 18,75 & 132 & 11,39 & 8,20 & 1,58 & 27,63 \\
\hline 23 & 10,04 & 7,23 & 1,53 & 23,75 & 78 & 10,54 & 7,79 & 1,52 & 21,75 & 133 & 11,08 & 8,89 & 1,17 & 26,75 \\
\hline 24 & 10,16 & 10,02 & 1,95 & 20,50 & 79 & 10,08 & 6,72 & 1,41 & 19,75 & 134 & 10,74 & 8,27 & 1,26 & 27,63 \\
\hline 31 & 10,02 & 6,91 & 1,42 & 20,00 & 86 & 10,94 & 6,84 & 1,78 & 18,75 & 141 & 9,83 & 8,19 & 0,77 & 21,00 \\
\hline 32 & 11,32 & 7,90 & 1,81 & 18,25 & 87 & 11,24 & 6,60 & 1,95 & 14,25 & 142 & 10,69 & 9,06 & 0,85 & 20,00 \\
\hline 33 & 10,55 & 7,88 & 1,18 & 20,50 & 88 & 11,23 & 8,85 & 1,19 & 34,00 & 143 & 9,81 & 8,12 & 0,82 & 17,50 \\
\hline 34 & 10,58 & 7,56 & 1,38 & 20,50 & 89 & 10,98 & 5,81 & 2,14 & 16,25 & 144 & 11,19 & 9,05 & 1,00 & 18,50 \\
\hline 35 & 10,91 & 7,92 & 1,42 & 21,50 & 90 & 11,57 & 8,96 & 1,29 & 20,50 & 145 & 10,44 & 8,81 & 0,85 & 17,50 \\
\hline 36 & 9,41 & 8,04 & 0,85 & 35,75 & 91 & 10,09 & 8,09 & 1,00 & 25,67 & 146 & 10,06 & 8,25 & 0,83 & 27,00 \\
\hline 37 & 10,05 & 6,74 & 1,74 & 18,25 & 92 & 10,03 & 7,41 & 0,88 & 33,50 & 147 & 10,14 & 7,11 & 1,45 & 26,50 \\
\hline 38 & 10,37 & 8,73 & 0,83 & 34,00 & 93 & 11,56 & 9,45 & 1,03 & 35,25 & 148 & 9,80 & 8,16 & 0,90 & 30,50 \\
\hline 39 & 12,42 & 8,72 & 1,81 & 20,00 & 94 & 12,23 & 6,46 & 2,82 & 16,75 & 149 & 9,64 & 6,82 & 1,40 & 38,50 \\
\hline 40 & 10,50 & 7,32 & 1,46 & 20,50 & 95 & 12,01 & 8,80 & 1,62 & 36,67 & 150 & 10,32 & 6,87 & 1,56 & 34,33 \\
\hline 41 & 9,89 & 7,44 & 1,44 & 23,25 & 96 & 11,73 & 8,51 & 1,60 & 19,25 & 151 & 11,55 & 8,21 & 1,62 & 3,67 \\
\hline 42 & 12,07 & 8,52 & 1,86 & 26,00 & 97 & 11,61 & 6,85 & 2,76 & 18,25 & 152 & 11,39 & 7,85 & 1,62 & 30,00 \\
\hline 43 & 11,33 & 8,49 & 1,44 & 20,75 & 98 & 11,42 & 7,23 & 2,46 & 15,00 & 153 & 11,65 & 8,71 & 1,31 & 29,33 \\
\hline 44 & 12,79 & 9,40 & 1,94 & 20,25 & 99 & 10,38 & 7,62 & 1,51 & 23,75 & 154 & 9,76 & 6,30 & 1,47 & 28,50 \\
\hline 45 & 13,01 & 10,31 & 1,74 & 20,25 & 100 & 10,33 & 9,51 & 0,91 & 40,50 & 155 & 10,16 & 8,45 & 0,82 & 18,00 \\
\hline 46 & 11,06 & 8,58 & 1,24 & 25,25 & 101 & 12,57 & 10,50 & 1,27 & 26,75 & 156 & 10,64 & 9,03 & 0,81 & 20,00 \\
\hline 47 & 12,05 & 8,41 & 1,87 & 26,00 & 102 & 10,97 & 7,35 & 1,92 & 20,75 & 157 & 9,97 & 8,18 & 0,97 & 19,00 \\
\hline 48 & 11,04 & 7,01 & 1,98 & 19,00 & 103 & 10,64 & 8,85 & 0,91 & 33,33 & 158 & 10,24 & 8,54 & 0,84 & 19,00 \\
\hline 49 & 12,32 & 8,51 & 1,70 & 19,50 & 104 & 11,10 & 8,86 & 1,51 & 41,50 & 159 & 11,42 & 9,29 & 1,07 & 23,00 \\
\hline 50 & 11,32 & 8,41 & 1,30 & 21,50 & 105 & 10,38 & 8,05 & 1,05 & 27,00 & 160 & 9,86 & 8,15 & 0,74 & 22,00 \\
\hline
\end{tabular}




\section{tabla 4. Parámetros geométricos medidos en diverSAS PLANTACIONES A CAÑAS GUADUA ANGUSTIFOLIA KUNTH}

\begin{tabular}{|c|c|c|c|c|c|}
\hline $\begin{array}{r}\text { NRO. DE } \\
\text { MUESTRA }\end{array}$ & $\begin{array}{r}\text { DIAMETRO } \\
\text { EXTERIOR (CM) }\end{array}$ & $\begin{array}{r}\text { S. ITERNUDO } \\
\text { (CM) }\end{array}$ & $\begin{array}{r}\text { NRO. DE } \\
\text { MUESTRA }\end{array}$ & $\begin{array}{r}\text { DIAMETRO } \\
\text { EXTERIOR (CM) }\end{array}$ & $\begin{array}{r}\text { S. ITERNUDO } \\
\text { (CM) }\end{array}$ \\
\hline 166 & 14,96 & 20,00 & 207 & 9,55 & 21,00 \\
\hline 167 & 11,14 & 18,00 & 208 & 11,46 & 20,00 \\
\hline 168 & 10,82 & 18,00 & 209 & 10,82 & 21,75 \\
\hline 169 & 14,96 & 20,00 & 210 & 10,82 & 22,00 \\
\hline 170 & 11,14 & 18,00 & 211 & 9,55 & 24,00 \\
\hline 171 & 10,19 & 20,00 & 212 & 9,87 & 17,50 \\
\hline 172 & 9,87 & 19,00 & 213 & 8,91 & 17,50 \\
\hline 173 & 10,19 & 23,00 & 214 & 9,23 & 18,50 \\
\hline 174 & 12,41 & 23,00 & 215 & 9,55 & 17,50 \\
\hline 175 & 10,82 & 20,00 & 216 & 10,82 & 17,00 \\
\hline 176 & 15,28 & 20,00 & 217 & 9,87 & 17,25 \\
\hline 177 & 10,50 & 20,00 & 218 & 9,55 & 19,00 \\
\hline 178 & 10,50 & 19,00 & 219 & 10,50 & 18,20 \\
\hline 179 & 11,14 & 23,00 & 220 & 9,55 & 18,83 \\
\hline 180 & 12,41 & 22,00 & 221 & 9,87 & 20,56 \\
\hline 181 & 10,50 & 20,00 & 222 & 11,14 & 26,50 \\
\hline 182 & 13,05 & 22,00 & 223 & 13,37 & 30,50 \\
\hline 183 & 11,46 & 20,00 & 224 & 10,82 & 35,00 \\
\hline 184 & 11,78 & 19,00 & 225 & 13,37 & 27,00 \\
\hline 185 & 13,05 & 22,00 & 226 & 14,64 & 27,50 \\
\hline 186 & 11,46 & 20,00 & 227 & 14,32 & 27,33 \\
\hline 187 & 11,78 & 19,00 & 228 & 11,14 & 36,50 \\
\hline 188 & 10,82 & 21,00 & 229 & 10,5 & 42,00 \\
\hline 189 & 10,19 & 22,00 & 230 & 12,41 & 38,50 \\
\hline 190 & 10,50 & 21,00 & 231 & 12,1 & 34,33 \\
\hline 191 & 13,05 & 21,00 & 232 & 11,78 & 30,67 \\
\hline 192 & 13,05 & 22,00 & 233 & 9,87 & 41,00 \\
\hline 193 & 12,73 & 19,00 & 234 & 12,41 & 36,67 \\
\hline 194 & 13,05 & 21,00 & 235 & 11,14 & 34,00 \\
\hline 195 & 12,73 & 20,00 & 236 & 11,78 & 40,50 \\
\hline 196 & 9,87 & 19,50 & 237 & 13,69 & 30,00 \\
\hline 197 & 12,41 & 23,00 & 238 & 12,73 & 29,33 \\
\hline 198 & 10,82 & 21,00 & 239 & 10,82 & 28,50 \\
\hline 199 & 9,87 & 21,50 & 240 & 14,32 & 26,86 \\
\hline 200 & 9,55 & 22,50 & 241 & 13,69 & 21,00 \\
\hline 201 & 11,14 & 22,00 & 242 & 13,05 & 21,00 \\
\hline 202 & 9,55 & 22,00 & 243 & 13,37 & 21,00 \\
\hline 203 & 12,10 & 22,00 & 244 & 13,69 & 21,00 \\
\hline 204 & 10,50 & 24,50 & 245 & 10,5 & 20,50 \\
\hline 205 & 9,55 & 20,50 & 246 & 10,82 & 20,00 \\
\hline 206 & 9,55 & 22,00 & & & \\
\hline
\end{tabular}

\section{S. Int (cm) - Esp (cm)}

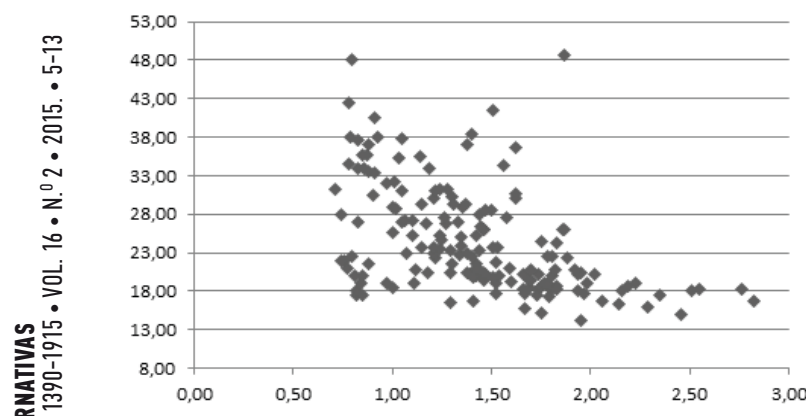

Figura 4. Variación de la longitud del entrenudo de la caña guadua vs. el espesor de la pared de la caña. A medida que disminuye la longitud del entrenudo disminuye el espesor de la pared de la caña
Análisis de las relaciones espesor y diámetro exterior (Esp/Dext), diámetro interior y diámetro exterior (Dint/Dext), diámetro medio $(\mathrm{Dm})$, diámetro exterior y longitud de entrenudo (Dext/S. Int).

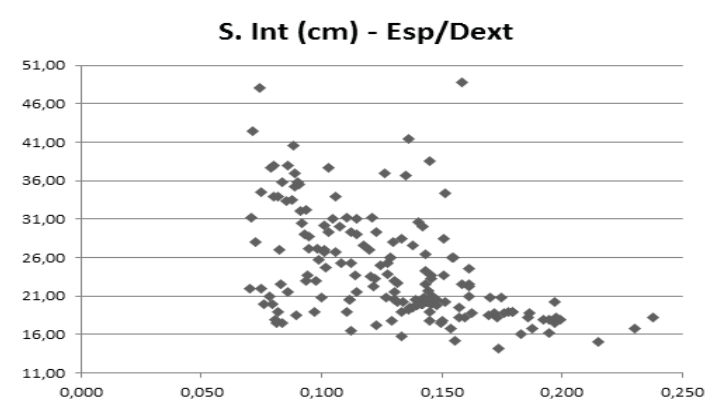

Figura 5. Variación de la longitud del entrenudo de la caña guadua vs. la relación (Espesor/Diámetro exterior). A medida que aumenta la relación (Esp/Dext) disminuye la longitud del entrenudo.

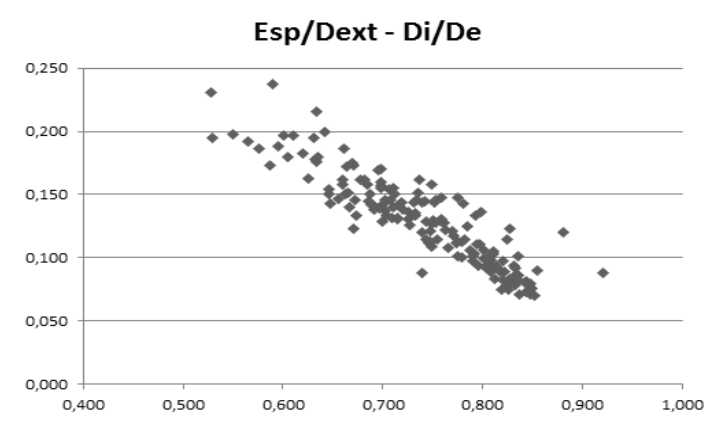

Figura 6. Variación de la relación (Espesor/Diámetro exterior) de la caña guadua vs. la relación (Diámetro interior/diámetro exterior). A medida que aumenta la relación (Dint/Dext) disminuye la relación (Esp/Dext).

Esp/Dext - Dext/S. Int

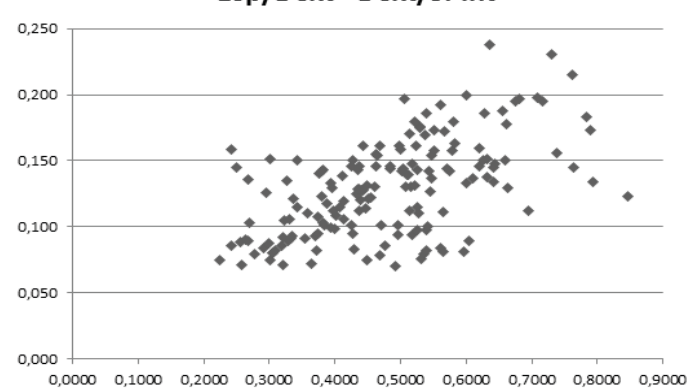

Figura 7. Variación de la relación (Espesor/Diámetro exterior) de la caña guadua vs. la relación (Diámetro exterior/longitud de entrenudo). A medida que aumenta la relación (Dext/S. Int) aumenta la relación (Esp/Dext). 
Algo muy importante a destacar es que la relación entre el espesor y el diámetro exterior (Esp/ Dext) permite encontrar relación con el resto de propiedades y relaciones geométricas de la caña guadua.

Haciendo un análisis estadístico de las propiedades geométricas de la caña guadua como el diámetro exterior, espesor y longitud del entrenudo. De los gráficos de las figuras 8, 9 y 10 se puede analizar que tan frecuente se puede encontrar con la medida específica de la propiedad que se desea.

En la figura 8 se observa que el rango de mayor probabilidad del diámetro exterior de una caña guadua se encuentra entre $9.803 \mathrm{~cm}$ y $11.651 \mathrm{~cm}$, siendo el valor promedio máximo de $10.697 \mathrm{~cm}$ la medida común más probable que tenga una caña guadua.

\section{TABLA 5. ANÁLISIS ESTADÍSTICO DEL DIÁMETRO EXTERIOR DE LAS CAÑAS GUADUA}

\begin{tabular}{l|r}
\hline Dext Media & $11.130 \mathrm{~cm}$ \\
\hline Desviación estándar (S) & $1.198 \mathrm{~cm}$ \\
\hline Dextk & $9.159 \mathrm{~cm}$ \\
\hline S/Dext Media & 0.108 \\
\hline
\end{tabular}

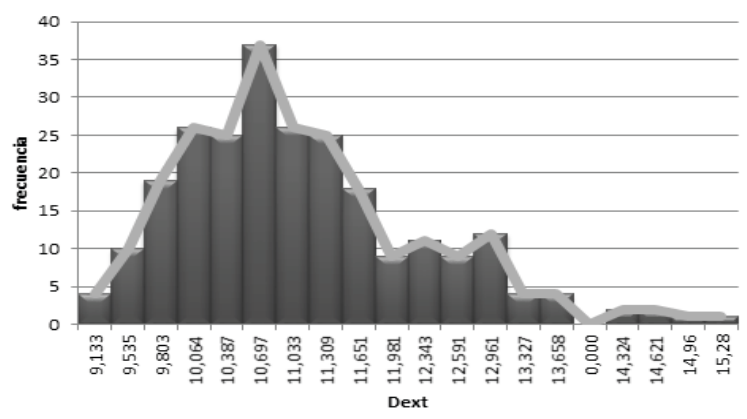

Figura 8. Análisis estadístico del diámetro exterior de la caña guadua.

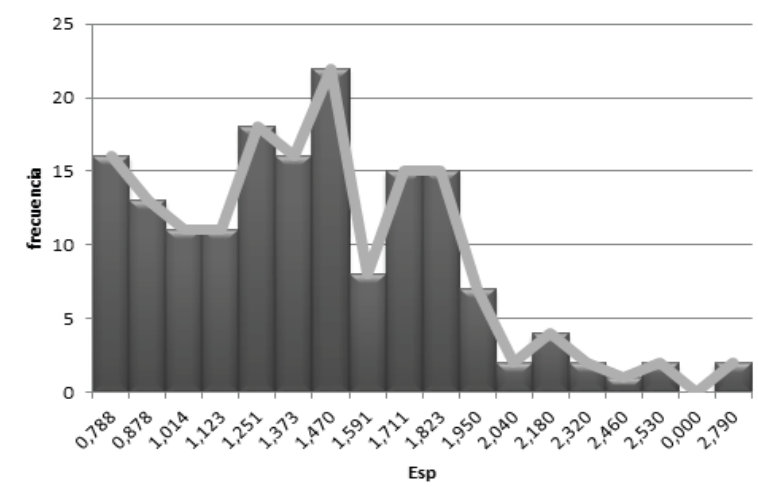

Figura 9. Análisis estadístico del espesor de la caña guadua.
En la figura 9 se observa que el rango de mayor probabilidad del espesor de la pared de una caña guadua se encuentra entre $0.788 \mathrm{~cm}$ y $1.823 \mathrm{~cm}$, siendo el valor promedio máximo de $1.47 \mathrm{~cm}$ la medida común más probable que tenga una caña guadua. En esta propiedad podemos encontrar mucha variación en los datos.

\section{TABLA 6. ANÁLISIS ESTADÍSTICO DEL ESPESOR DE LA PARED DE LAS CAÑAS GUADUA}

\begin{tabular}{lr} 
Esp Media & $1.420 \mathrm{~cm}$ \\
Desviacion estandar (S) & $0.440 \mathrm{~cm}$ \\
\hline Espk & $0.697 \mathrm{~cm}$ \\
S/Esp Media & 0.310 \\
\hline
\end{tabular}

En la figura 10 se observa que el rango de mayor probabilidad de la longitud de entrenudos de una caña guadua se encuentra entre $18.537 \mathrm{~cm}$ y $22.048 \mathrm{~cm}$, siendo el valor promedio máximo de $20.354 \mathrm{~cm}$ la medida común más probable que tenga una caña guadua.

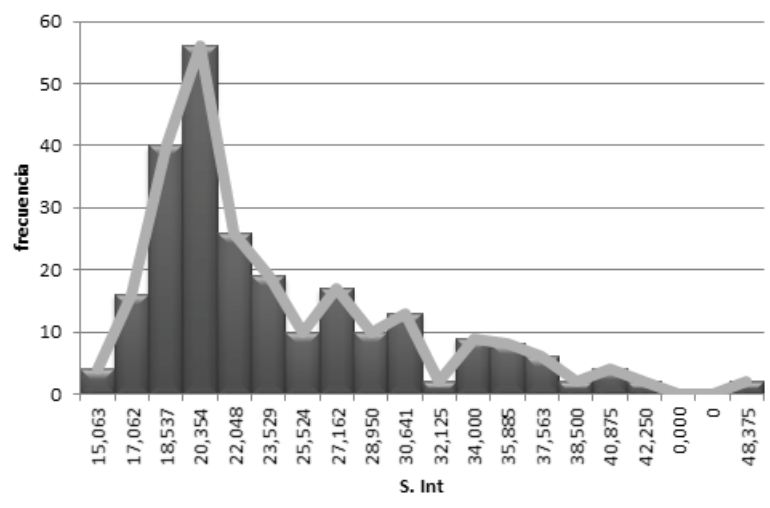

Figura 10. Análisis estadístico de la longitud de entrenudo de la caña guadua.

TABLA 7. ANÁLISIS ESTADÍSTICO DE LA LONGITUD DE ENTRENUDO DE LAS CAÑAS GUADUA.

\begin{tabular}{lr} 
S. Int Media= & $24.163 \mathrm{~cm}$ \\
Desviación estándar= & $6.659 \mathrm{~cm}$ \\
S. Intk= & $10.178 \mathrm{~cm}$ \\
S/S. Int Media= & 0.276 \\
\hline
\end{tabular}

Haciendo un análisis estadístico de las relaciones geométricas de la caña guadua como el espesor y diámetro exterior (Esp/Dext), diámetro interior y diámetro exterior (Dint/Dext), diámetro exterior y longitud de entrenudo (Dext/S. Int). 


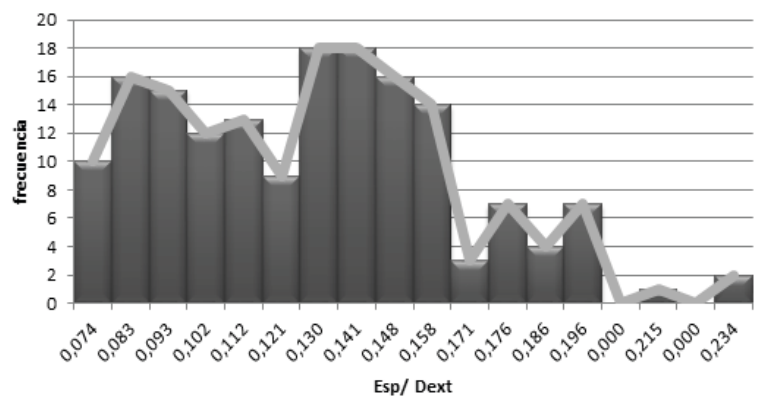

Figura 19. Análisis estadístico de la relación (Espesor/Diámetro exterior).

TABLA 8. ANÁLISIS ESTADÍSTICO DE LA RELACIÓN (ESP/DEXT) DE LAS CAÑAS GUADUA

\begin{tabular}{ll} 
Esp/ Dext Media $=$ & 0,129 \\
Desviación Estándar (S) & 0,036 \\
(Esp/Dext) $k=$ & 0,070 \\
S/Esp/Dext Media $=$ & 0,280 \\
\hline
\end{tabular}

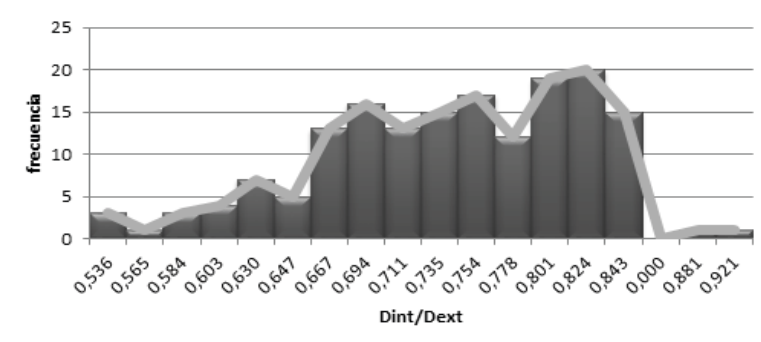

Figura 20: Análisis estadístico de la relación (Diámetro interior/Diámetro exterior).

\begin{tabular}{|c|c|}
\hline Dint/Dext Media & 0,741 \\
\hline Desviación Estándar (S) & 0,078 \\
\hline (Dint/Dext)K & 0,613 \\
\hline S/Dint/Dext Media & 0,105 \\
\hline
\end{tabular}

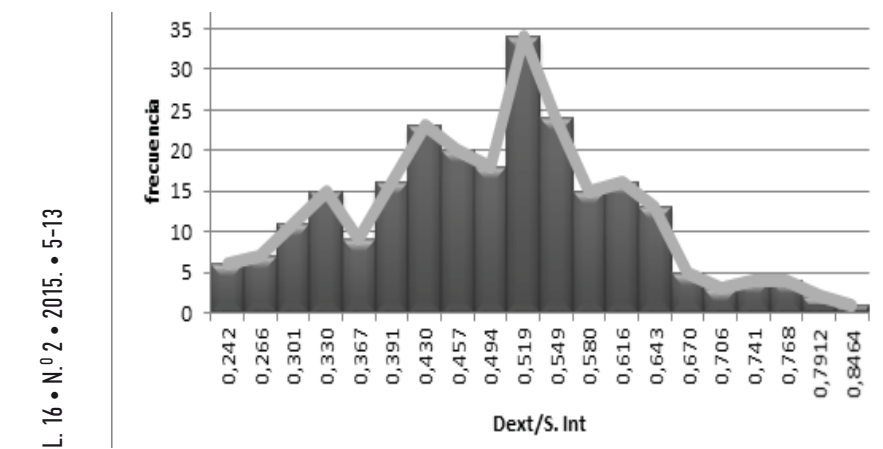

Figura 21. Análisis estadístico de la relación (Diámetro exterior/Longitud de entrenudo).
TABLA 10. ANÁLISIS ESTADÍSTICO DE LA RELACIÓN (DEXT/S. INT) DE LAS CAÑAS GUADUA

\begin{tabular}{ll} 
Dext/S. Int Media & 0.491 \\
\hline Desviación estándar (S) & 0.126 \\
\hline (Dext/S. Int) & 0.284 \\
\hline S/Dext/S. Int Media & 0.257 \\
\hline
\end{tabular}

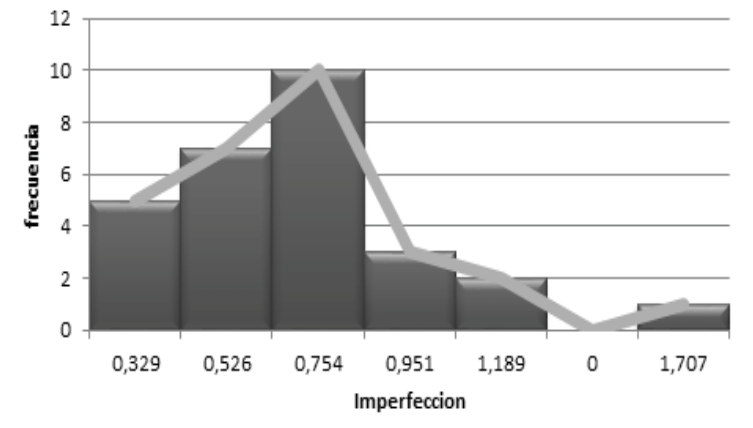

Figura 22. Análisis estadístico de la imperfección vertical de las cañas guadua.

TABLA 11. ANÁLISIS ESTADÍSTICO DE LA IMPERFECCIÓN VERTICAL DE LAS CAÑAS GUADUA

\begin{tabular}{ll}
\hline Imperfección Media & 0.707 \\
\hline Desviación Estándar (S) & 0,316 \\
\hline Imperfección k & 0.187 \\
\hline Imperfección Media & 0.447 \\
\hline
\end{tabular}

\section{ANÁLISIS ESTADÍSTICO DE LA IMPERFECCIÓN VERTICAL DE} LAS CAÑAS GUADUA

Se determinó de la población de 28 muestras que el porcentaje de imperfección máximo que existió fue de $1.7 \%$, el cual es casi $2 \%$, el límite para el cual se puede tener una influencia notable de las imperfecciones en fenómenos como pandeo de columnas esbeltas. El promedio fue de $0.7 \%$, el cual es bastante aceptable.

DISCUSIÓN

Delanálisis hechoa las propiedades geométricas de la caña guadua se resalta que cuando se tiene un diámetro externo grande, se tendrá un diámetro interior mayor, un espesor mayor, una longitud de entrenudo menor. Además con un espesor de la pared de la caña guadua mayor se tendrá una relación entre el diámetro exterior y la longitud del entrenudo mayor, una relación entre el diámetro interior y el diámetro exterior menor, y una relación entre el espesor de la pared de la caña y el diámetro exterior de la caña mayor. 


\begin{tabular}{|c|c|c|c|c|c|c|c|}
\hline 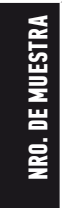 & 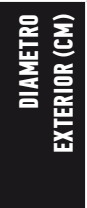 & 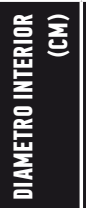 & $\begin{array}{l}\text { 둘 } \\
\text { 営 } \\
\text { 兽 }\end{array}$ & 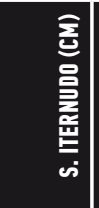 & 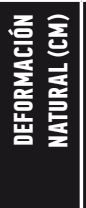 & 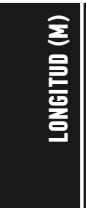 & \\
\hline 1 & 9.17 & 5,92 & 1.41 & 16.75 & 5.14 & 6.14 & \\
\hline 2 & 9.83 & 5,66 & 1.83 & 18.2 & 10.41 & 6.1 & 1.70 \\
\hline 3 & 9.78 & 7.63 & 1.4 & 22.5 & 4.08 & 6.1 & 0.669 \\
\hline 4 & 12.2 & 8.37 & 1.77 & 19 & 7.09 & 6.14 & 1.115 \\
\hline 5 & 10.03 & 8.39 & 0.71 & 31.25 & 4.48 & 6 & 0.747 \\
\hline 6 & 10.97 & 6.53 & 2.06 & 16.75 & 2.8 & 6.06 & 0.462 \\
\hline 7 & 13.57 & 10.08 & 1.97 & 17.75 & 4.16 & 5.97 & 0.697 \\
\hline 8 & 10.22 & 7.92 & 1.51 & 19.75 & 5.93 & 6.03 & 0.983 \\
\hline 9 & 9.91 & 5.99 & 1.78 & 19 & 2.83 & 5 & 0.566 \\
\hline 10 & 10.82 & 8.49 & 1.35 & 25 & 3.45 & 6.2 & 0.556 \\
\hline 11 & 10.6 & 8 & 1.21 & 23.75 & 5.87 & 6.08 & 0.965 \\
\hline 12 & 10.57 & 7.5 & 1.39 & 20.25 & 2.34 & 6 & 0.390 \\
\hline 13 & 12.92 & 7.1 & 2.55 & 18.25 & 4.3 & 6.08 & 0.811 \\
\hline 14 & 11.14 & 9.21 & 1.37 & 2925,00 & 4.94 & 6.21 & 0.795 \\
\hline 15 & 11.61 & 9.61 & 0.93 & 38 & 1.36 & 6.2 & 0.219 \\
\hline 16 & 12.87 & 10.38 & 1.3 & 30.25 & 7.34 & 6 & 1.223 \\
\hline 17 & 12.3 & 9.72 & 1.25 & 24.75 & 4.6 & 6.2 & 0.742 \\
\hline 18 & 10.1 & 5.71 & 194 & 18 & 3.77 & 6.1 & 0.618 \\
\hline 19 & 11.365 & 8.45 & 1.46 & 16.075 & 2.78 & 3.8 & 0.732 \\
\hline 20 & 11.215 & 8.915 & 1.24 & 31.25 & 1.01 & 4.065 & 0.248 \\
\hline 21 & 11.84 & 9.26 & 1.355 & 29 & 1.95 & 3.65 & 0.534 \\
\hline 22 & 10.45 & 8.615 & 0.825 & 37.625 & 1.9 & 3.72 & 0.511 \\
\hline 23 & 11.595 & 9.365 & 1.1 & 27.125 & 3.18 & 3.78 & 0.841 \\
\hline 24 & 11,39 & 8.2 & 1.575 & 27.625 & 1.64 & 4 & 0.410 \\
\hline 25 & 11.08 & 8.885 & 1.17 & 26.75 & 1.69 & 3.87 & 0.437 \\
\hline 26 & 10.735 & 8.27 & 1.26 & 27.625 & 3.34 & 3.7 & 0.903 \\
\hline 27 & 10.53 & 8.015 & 1.345 & 23.875 & 2.5 & 3.76 & 0.665 \\
\hline 28 & 10.86 & 8.775 & 1.065 & 27.875 & 1.44 & 3.8 & 0.379 \\
\hline
\end{tabular}

Se determinó la relación entre el espesor de la pared de la caña y el diámetro exterior de la caña (Esp/Dext), como la relación más importante, y que más ayuda puede dar en la determinación del resto de propiedades geométricas. Es muy importante que esta relación geométrica sea alta para poder tener una caña con propiedades geométricas adecuadas.

En la tabla 2 se realizó el cálculo de las propiedades físicas y estructurales para el valor característico de la relación Esp/Dext. Estas son las propiedades mínimas que va a tener una caña guadua, en las que se tiene un fratil de 5\% de probabilidad, que las propiedades puedan ser menor que las que se determinan en las tablas. Es decir tenemos un 95\% de seguridad de que las propiedades geométricas, físicas y estructurales van a ser mayores.

Con la metodología utilizada se puede considerar la caña como un perfil de tipo industrial. Por lo tanto, en el caso del diseño de estructuras el diseñador indica solo el diámetro exterior, los valores característicos debe determinarlo asegurando una zona fiable para la seguridad de la estructura. La carga y reacciones que interesan a la estructura deben ser relacionados como se una normalmente en el diseño de estructuras.

AGRADECIMIENTOS

A la Universidad Católica de Santiago de Guayaquil por permitir a este equipo de profesionales realizar esta investigación y colaborar con todo lo que se necesitó para el éxito de la determinación de los resultados.

\section{REFERENCIAS BIBLIOGRÁFICAS}

La Tegola, A., Mera, W. (2012). Diseño de las estructuras de hormigón armado a los estados límites. Universidad Católica de Santiago de Guayaquil.

Córdova, P. (2014). Obtención de las Propiedades mecánicas y estructurales de la caña Guadua Angustifolia Kunth del Ecuador. Trabajo de Grado, facultad de Ingeniería. Universidad Católica de Santiago de Guayaquil. 